\title{
A Note on Entanglement Entropy, Coherent States and Gravity
}

\author{
Madhavan Varadarajan ${ }^{1, *}$ \\ ${ }^{1}$ Raman Research Institute, Bangalore 560080, India
}

\begin{abstract}
The entanglement entropy of a free quantum field in a coherent state is independent of its stress energy content. We use this result to highlight the fact that while the Einstein equations for first order variations about a locally maximally symmetric vacuum state of geometry and quantum fields seem to follow from Jacobson's principle of maximal vacuum entanglement entropy, their possible derivation from this principle for the physically relevant case of finite but small variations remains an open issue. We also apply this result to the context of Bianchi's identification, independent of unknown Planck scale physics, of the first order variation of BekensteinHawking area with that of vacuum entanglement entropy. We argue that under certain technical assumptions this identification seems not to be extendible to the context of finite but small variations to coherent states.

Our particular method of estimation of entanglement entropy variation reveals the existence of certain contributions over and above those of References [2, 5]. We discuss the sense in which these contributions may be subleading to those in References [2, 5].
\end{abstract}

\section{INTRODUCTION}

In his path breaking work of 1995, Jacobson [1] derived the classical Einstein equations as conditions of thermal equilibrium in local Rindler Wedges at every point in spacetime. Recently, Jacobson [2] provided a deeper implementation of his ideas by moving his analysis from the setting of local Rindler wedges to causal domains of small spherical regions about every spacetime point. Instead of classical matter flux through local Rindler horizons, he considered quantum matter fields restricted to these causal domains and thereby obtained the semiclassical Einstein equations as equilibrium conditions on the entanglement entropy of the quantum matter in these causal domains. More in detail, Jacobson proposed that this entanglement entropy is maximal when the local geometry in the causal domain is maximally symmetric and the quantum fields are in the associated maximally symmetric vacuum. This implies that first order variations about this maximal entropy configuration must vanish where, by first order variation we mean the derivative with respect to a parameter labelling the state and geometry, evaluated at the maximally symmetric configuration. Jacobson showed that this implication of maximal vacuum entropy was equivalent to the satisfaction of the Einstein equations for such first order variations. A key feature of his analysis is the emergence of the stress energy of the matter field through quantum entanglement via the so called First Law of Entanglement Entropy [3]. This Law, under certain conditions [2, 3], relates first order variations of stress energy expectation value to those of entanglement entropy.

Whereas first order variations involve limiting behaviour under infinitesmal changes, for

*Electronic address: madhavan@rri.res.in 
applicability to physical situations one would hope that the analysis of Reference [2] could be extended from the case of infinitesmal variations to finite but small variations. In this note we recall that there exist, at least for free field theories, quantum states which are finite but small variations of the vacuum and whose entanglement entropy is independent of their stress energy content. This fact seems to be in tension with the non-trivial dependence of stress energy on entropy suggested by the First Law of Entanglement Entropy, and hence with Jacobson's results. As we shall see, the resolution of this apparent tension is that for coherent states which are finite but small variations about the vacuum, the stress energy expectation value vanishes to first order in the smallness parameter. Thus, to first order in the smallness parameter, the variation of the entropy and that of the stress energy both vanish. Since the First Law of Entanglement Entropy as well as Jacobson's derivation are first order analyses, no contradiction arises. Nevertheless, as the work of Ford and Kuo [4] indicates, quantum matter in a coherent state provides a setting in which we do expect the semiclassical Einstein equations to hold irrespective of the smallness of the stress energy content of the coherent state. Hence a generalization of the considerations of Reference 2] to coherent states which are finite but small variations of the vacuum constitutes an (extremely interesting) open issue.

Our general remarks pertain to any setting in which the First Law of Entanglement Entropy is used to derive gravitational consequences of entanglement entropy variation. Another such setting is that of Bianchi's intriguing derivation of the first order variation of Bekenstein- Hawking entropy from the first order variation of entanglement entropy of quantum states across Causal Horizons [5] (see also Bianchi and Satz [6]) without recourse to UV physics. Once again, for applicability to physical situations we would like to enquire as to whether the considerations of Reference [5] also hold for finite but small variations. In this regard we shall argue that this derivation (modulo some technical assumptions) does not seem to be extendible to the context of coherent states which constitute finite but small variations of the vacuum.

The main purpose of this work is to detail the observations of the previous paragraphs. For concreteness we shall restrict attention to matter constituted by a single free scalar field. The layout of this note is then as follows. In section 2 we recall that the entanglement entropy of any coherent state of a free quantum field in a causal domain is the same as the entanglement entropy of the free field vacuum state in the causal domain. Next, we show that in a coherent state which is a finite, small variation about the vacuum, the stress energy expectation value is non-trivial only at second order in the smallness parameter.

In section 3 we compute the first order variation of entanglement entropy and stress energy under variations of geometry and state around their maximally symmetric vacuum configurations. Our particular method of computation yields certain contributions to these variations over and above those which appear in Reference [2]. We discuss the sense in which these contributions maybe subleading to those in [2]. From the considerations of [2], it then follows that their neglect reproduces the derivation of the Einstein equations for such (infinitesmal) variations.

In Section 4 we estimate the change in entanglement entropy $S$ and stress energy $T_{a b}$ under finite but small variations of geometry and state. More in detail, we consider a 1 parameter family of geometries and states and estimate $S, T_{a b}$ at finite, small values of the parameter relative to their values at the origin in the context of a Taylor approximation. We use the results of Sections 2 and 4 in sections 5 and 6 . Hence our conclusions in those sections are contingent on our assumed existence of the Taylor approximation of section 4 . 
In section 5 we confront Jacobson's principle of maximal vacuum entanglement entropy [2] with coherent states which are finite but small variations of the vacuum. We argue that while the existence of a derivation of the Einstein equations from Jacobson's principle of maximal vacuum entanglement entropy for variations to such coherent states remains an open issue, the Einstein equations may be consistent with this principle. ${ }^{1}$

In section 6, we turn our attention to the considerations of Reference [5] and argue that (under certain technical assumptions) Bianchi's ideas relating first order variations of Bekenstein Hawking area with those of entanglement entropy using the quantum Einstein equations do not seem to be extendible to the case of coherent states which are finite but small variations of the vacuum.

\section{ENTANGLEMENT ENTROPY AND STRESS ENERGY OF COHERENT STATES.}

The result that the entanglement entropy of any coherent state is the same as the entanglement entropy of the vacuum state has been derived in various contexts [7 $[9]$. While Reference [7] focuses on $1+1$ dimensions and uses the conformal flatness of two dimensional geometries, the considerations of References [8, 9] are restricted to flat spacetimes. As in References [7 9], we shall restrict attention in this paper to free scalar field matter.

The underlying reason for the equality of coherent state and vacuum entanglement entropies as succintly stated in Reference [8] is that any coherent state can be written as a particular unitary transformation of the vacuum which factorizes as the product of a pair unitary transformations one depending only on the degrees of freedom being traced over and the other, on the remaining degrees of freedom. The equality of entanglement entropies then follows from the invariance of the tracing operation under such unitary maps. More in detail, consider a free (real) scalar field $\Phi$ on a 4 dimensional flat spacetime with inertial coordinates $(\vec{x}, t)$. Consider a coherent state $|H\rangle$ which is modelled on a classical scalar field solution $H(\vec{x}, t)$. It is straightforward to verify that

$$
|H\rangle=\hat{U}(e, f)|0\rangle,
$$

where

$$
\hat{U}(e, f):=e^{i \int_{R^{3}} d^{3} x f(\vec{x}) \hat{\phi}(\vec{x})-e(\vec{x}) \hat{\pi}(\vec{x})} .
$$

Here, the integral is over some constant $t$ slice, $\hat{\phi}(\vec{x}), \hat{\pi}(\vec{x})$ are the scalar field and conjugate momentum operators on this slice and $e(\vec{x}), f(\vec{x})$ are the scalar field and conugate momentum induced on the slice by the spacetime solution $H(\vec{x}, t)$.

Let $\varnothing$ be a 3 dimensional region on this slice. The entanglement entropy of a state is the Von Neumann entropy of the density matrix obtained by tracing over the scalar field degrees of freedom in the complement of $\Omega$. Let us label quantities defined within $\Omega$ by the subscript $\varnothing$ and those defined in $R^{3}-\Omega$ by $\Omega^{c}$. For example, $\operatorname{Tr}_{\Omega^{c}}$ refers to a trace over degrees of freedom in $\Omega^{c}$. The density matrix for $|H\rangle$ is then $\hat{\rho}(H)=\operatorname{Tr}_{\Omega^{c}}(|H\rangle\langle H|)$ and that for the vacuum is $\hat{\rho}(0)=\operatorname{Tr}_{\Omega^{c}}(|0\rangle\langle 0|)$. Denote the corresponing entanglement entropies by $S(H)=-\operatorname{Tr}_{\varnothing}(\hat{\rho}(H) \ln \hat{\rho}(H)), S(0)=-\operatorname{Tr}_{\varnothing}(\hat{\rho}(0) \ln \hat{\rho}(0))$.

\footnotetext{
${ }^{1}$ This consistency was anticipated by Jacobson in [2]
} 
We have the factorization:

$$
\hat{U}(e, f)=\hat{U}_{\Omega^{c}}(e, f) \hat{U}_{\varnothing}(e, f)
$$

with

$$
\hat{U}_{\varnothing}(e, f)=e^{i \int_{\varnothing} f(x) \phi(x)-e(x) \pi(x)}, \hat{U}_{\Omega^{c}}(e, f)=e^{i \int_{\Omega^{c}} f(x) \phi(x)-e(x) \pi(x)} .
$$

It follows that

$$
\begin{aligned}
\hat{\rho}(H) & =\operatorname{Tr}_{\Omega^{c}}\left(\hat{U}_{\Omega^{c}}(e, f) \hat{U}_{\varnothing}(e, f)|0\rangle\langle 0| \hat{U}_{\varnothing}(e, f)^{\dagger} \hat{U}_{\Omega^{c}}(e, f)^{\dagger}\right) \\
& =\operatorname{Tr}_{\Omega^{c}}\left(\hat{U}_{\varnothing}(e, f)|0\rangle\langle 0| \hat{U}_{\varnothing}(e, f)^{\dagger}\right)=\hat{U}_{\varnothing}(e, f) \operatorname{Tr}_{\Omega^{c}}(|0\rangle\langle 0|) \hat{U}_{\varnothing}(e, f)^{\dagger},
\end{aligned}
$$

where we have used the invariance of the Trace under unitary tranformations, and the fact that $U_{\varnothing}(e, f)$ acts as the identity operator on the degrees of freedom in $\Omega^{c}$ in the last line. The equality of $S(H)$ and $S(0)$ then follows, once again, from the invariance of the Trace under unitary transformations.

While equations (2.3) - (2.6) exhibit the simple underlying reason for the equality of $S(H)$ and $S(0)$, the equations themselves are UV divergent and need to be regulated because of the sharp boundary between $\varnothing$ and $\Omega^{c}$. The sharp boundary renders the calculations UV divergent in two ways. The first is the well known UV divergence of $S(0)$ itself. The second is that for $(e, f)$ which are non-vanishing on this boundary, the exponents in equation (2.4) are not well defined self adjoint operators. For concreteness let us use a lattice regulator as in Reference [9] to regulate the first divergence. The second one then automatically disappears because the integrals of quantum fields over regions in the exponents of (2.4) become sums of quantum mechanical degrees of freedom over lattice sites and the exponents are thereby rendered mainfestly well defined. Since both divergences spring from the sharpness of the boundary, we expect that any UV regulated calculation which effectively removes the sharpness of this boundary makes equations (2.3) - (2.6) well defined and establishes the equality of $S(H)$ and $S(0)$.

Next we turn to the (flat spacetime) normal ordered stress energy tensor. It is easy to check that the normal ordered two point function in the coherent state $|H\rangle$ evaluates to

$$
\left\langle H\left|: \hat{\Phi}(\vec{x}, t) \hat{\Phi}\left(\vec{x}^{\prime}, t^{\prime}\right):\right| H\right\rangle=H(\vec{x}, t) H\left(\vec{x}^{\prime}, t^{\prime}\right)
$$

and the stress energy tensor expectation value is then exactly that of the classical solution $H(\vec{x}, t)$. We are interested in coherent states which are first order departures from the vacuum. Since the free field equations are linear, if $H$ is a solution with initial data $(e, f)$ as above, then $\delta H=\delta \times H$ is a solution with initial data $(\delta e, \delta f)$. Accordingly for some small positive parameter $\delta$ we have

$$
|\delta H\rangle=e^{i \delta \int_{R^{3}} d^{3} x f(\vec{x}) \hat{\phi}(\vec{x})-e(\vec{x}) \hat{\pi}(\vec{x})}|0\rangle .
$$

It is straightforward to expand the exponential out and verify that this state is a first order (in $\delta$ ) variation of the vacuum state as desired. It also follows from equation (2.7) that the normal ordered stress energy expectation value is of second order in $\delta$.

Our considerations so far have been in the context of flat spacetimes. In the appendix we define coherent states on curved spacetimes through an appropriate generalization of equation (2.1) and derive the curved spacetime generalizations of equations (2.3)- (2.8). While 
a detailed regulation of the UV divergences in the curved spacetime analogs of equations (2.3) - (2.6), is out of the scope of this work, the general discussion above of the softening of the sharp boundary via UV regulation indicates that the equality of vacuum and coherent state entanglement must continue to hold in the curved spacetime context.

\section{FIRST ORDER VARIATION OF ENTANGLEMENT ENTROPY AND STRESS ENERGY}

The entanglement entropy $S$ for degrees of freedom restricted to the spatial region $B$ and the stress energy expectation value $T_{a b}$ on $B$ depend on the spacetime geometry $g$ and the quantum state of the field $\psi$ in the vicinity of $B$. We indicate this dependence of $S, T_{a b}$ through the notation $S(g, \psi), T_{a b}(g, \psi)$. Let $\lambda$ be a real parameter taking values in some small interval about the origin. Let $B(\lambda)$ be the spatial region of interest. Let $g(\lambda)$ be the spacetime metric in the vicinity of $B(\lambda)$ and let $\psi(\lambda)$ be the free scalar field state on $B(\lambda)$. In Reference [2], $B(\lambda)$ denotes a small fixed volume geodesic ball (with respect to the metric $g(\lambda))$ around a spacetime point $p$. We use the diffeomorphism freedom to identify $B(\lambda)$ with $B(0):=B$. Finally, let $(\psi(0), g(0))$ be the maximally symmetric vacuum configuration.

The first order variation of any functional $A(g, \psi)$ is defined to be $\left.\left(\frac{d}{d \lambda} A(g(\lambda), \psi(\lambda))\right)\right|_{\lambda=0}$ The computation of the derivative assumes that there is some way of comparing the pair $(g(\lambda), \psi(\lambda)$ with the pair $(g(0), \psi(0))$ in an arbitrarily small neighbourhood of the origin. Since we are not aware of an explicit way of making such a pairwise comparision, we shall assume that we can independently vary $g$ and $\psi$ in the computation of this derivative. Thus we compute the derivative as a sum of two partial derivatives, one obtained by holding the geometry $g$ fixed at $g(0)$ and varying the state $\psi$ and the other by holding the state $\psi$ fixed at $\psi(0)$ and varying the geometry $g$. This in turn requires that we are able to compare the state $\psi(\tau)$ with $\psi(0)$ independent of the geometry $g(\lambda)$ and vice versa when $\lambda, \tau$ vary over some arbitrarily small neighbourhood of the origin. In particular, this implies that we are able to identify the same state on different geometries in an arbitrarily small neighbourhood of parameter space. We shall assume that it is possible for this identification to be made in such a way that the fixed state is Hadamard on these different geometries.

More precisely, we consider a 2 parameter family of states and geometries $(g(\lambda), \psi(\tau))$ with $\psi(\tau)$ assumed to be Hadamard with respect to the spacetime geometry $g(\lambda)$ in the vicinity of $B$ and in a small neighbourhood of parameter space around $\lambda=0=\tau$. It then follows that the first order variations of $S, T_{a b}$ evaluate to

$$
\begin{gathered}
\left(\frac{d S(g(\lambda), \psi(\lambda))}{d \lambda}\right)_{\lambda=0}=\left(\frac{\partial S(g(\lambda), \psi(0))}{\partial \lambda}\right)_{\lambda=0}+\left(\frac{\partial S(g(0), \psi(\tau))}{\partial \tau}\right)_{\tau=0} \\
\left(\frac{d T_{a b}(g(\lambda), \psi(\lambda))}{d \lambda}\right)_{\lambda=0}=\left(\frac{\partial T_{a b}(g(\lambda), \psi(0))}{\partial \lambda}\right)_{\lambda=0}+\left(\frac{\partial T_{a b}(g(0), \psi(\tau))}{\partial \tau}\right)_{\tau=0}
\end{gathered}
$$

Jacobson's proposal of maximal vacuum entanglement implies that the right hand side of equation(3.1) vanishes. The second term in equation (3.1) concerns the contribution obtained by varying the state but keeping the geometry fixed. Jacobson refers to this term as the IR contribution because it is independent of the UV physics which regulates the divergence in the entropy arising from short distance correlations close to the entangling surface. The assumed Hadamard behaviour of all states considered implies that the fixed 
geometry contribution of the second term is expected to be finite and dependent only on the IR physics. This is indeed true and the First Law of Entanglement entropy reexpresses this term as $1 / \hbar$ times (an integral of) the second term of equation (3.2). ${ }^{2}$

The first term in equation (3.1) corresponds to the contribution from the fixed state while varying the geometry. This is called the UV term in [2]. Since the area of the entangling surface changes when the metric is varied, and since the UV divergence of the entropy is expected, to leading order in the UV cutoff, to scale as the area one assumes that this term has a UV contribution corresponding to the variation of the area. However, in principle, there may also be additional IR contributions to the entropy which depend on the precise identification of the unvaried (vacuum) state as a state on the varied geometry.

If these IR contributions can be neglected, the argumentation of [2] holds whereby the UV contribution is related to the $1 / \hbar$ times ' $G_{a b}$ ' term and the remaining IR contribution, as mentioned above, to $1 / \hbar$ times the second term in the ' $T_{a b}$ ' equation (3.2). The Einstein equations for first order variations would then ensue if we could neglect the first term in equation (3.2) compared to the second term in that equation. Once again whether we can do this depends on the manner in which the unperturbed state is identified as a state on the perturbed spacetime. To summarise: If we assume that the geometry and state can be varied independently, we are able to obtain the Einstein equations for first order variations from equations (3.1) and (3.2) in conjunction with the principle of maximal vacuum entanglement only if the identification of the unvaried state on the varied geometry is such that we are able to neglect the first term of (3.2) as well as the IR contribution to the first term of (3.1).

Recall that the need to identify state spaces for the varied and unvaried geometries stems from our (current) inability to make a pairwise comparion between $(g(\lambda), \psi(\lambda))$ and $(g(0), \psi(0))$. If we could make such a pairwise comparision, the need to identify $\psi(0)$ as a state on the geometry $g(\lambda)$ would not be necessary. This suggests that our final conclusions should be independent of the way in which we identify state spaces on the varied and unvaried spacetime geometries. We also note that there is no canonical way to make this identification for generic variations of the geometry due to the generic lack of any (conformal) symmetry of the varied geometry. This suggests that we try to make this identification in such a way that the first term of (3.2) and the IR contribution to the first term of (3.1) can indeed be neglected.

In order to see one particular way in which these sort of 'identification dependent' contributions could be negligible, we present an argument suitably adapted here from Hawking[13] which suggests that for any physically reasonable identification the first term in equation (3.2) can be neglected compared to the second. The argument is as follows. The metric $g(\delta)$ at parameter value $\lambda=\delta$ is a small variation of $g(0)$ for $\delta<<1$ and we write it as $g(\delta)=g(0)+\delta g$. For modes which are high frequency with respect to the curvature scale, we do not expect a significant change in the annihilation- creation operators on the spacetime geometry $g(0)+\delta g$ relative to the annihilation- creation operators on $g(0)$. This is consistent with the assumed Hadamard behavior of the states. For modes which have a frequency comparable or less than the curvature scale, we expect that the annihilation- creation operators for the spacetime $g(0)+\delta g$ are modified by order $\delta$ relative to the annihilation- creation operators for $g(0)$. This results in a modification of order $\delta$ in the number operator for these modes. Approximating the mode density by the flat spacetime mode density $\omega^{2} d \omega$ of the

\footnotetext{
2 This is true for conformal matter, for the non-conformal case we assume Jacobson's conjecture [2] holds. See also Reference [10] in this regard.
} 
number of modes per unit volume at frequency $\omega$, we obtain a particle number density of the order of $\delta L^{-3}$ where the curvature scale of $g$ is $L^{-2}$. This in turn leads to an uncertainty of the energy per unit volume of order $\hbar \delta L^{-4}$, which is first order in $\hbar$. This argument (in the limit that $\delta \rightarrow 0$ ) suggests that the first term in equation (3.2) is of order $\hbar$. Since the stress energy in the Einstein equations is expected to be independent of $\hbar$, the first term of (3.2) is subleading in $\hbar$ and can be neglected.

It would be good to have a similar argument for the neglect of any IR contributions to the first term in equation (3.1). The argument above indicates that the uncertainty in the number of particles per unit volume is $\delta L^{-4}$ (which is of order $\hbar^{0}$ ) but we are not sure as to how this could be used to conclude that the additional IR corrections to the entropy arising from this first term are also of order $\hbar^{0}$.

\section{FINITE VARIATIONS OF ENTANGLEMENT ENTROPY AND STRESS ENERGY}

In this section we estimate the change in $S, T_{a b}$ under joint variations of geometry and state which are finite and small. Thus we are interested in the estimation of $S(g(\delta), \psi(\delta))-$ $S(g(0), \psi(0))$ and $T_{a b}(g(\delta), \psi(\delta))-T_{a b}(g(0), \psi(0))$ for $\delta<<1$. Similar to the previous section, we assume that the metric and state can be independently varied and that for $\tau, \lambda$ in a small neighbourhood of the origin an identification, of the state $\psi(\tau)$ on the spacetime geometry $g(\lambda)$ in the vicinity of the spatial region of interest $B$, has been made in such a way as to preserve its Hadamard property. Further, we shall assume that $S, T_{a b}$ admit a Taylor approximation to second order in the smallness parameter $\delta$.

The expansion of the entanglement entropy to second order in $\delta$ is then:

$$
\begin{aligned}
S(g(\delta), \psi(\delta))-S(g(0), \psi(0)) & =\Delta S^{(1)}+\Delta S^{(2)} \\
\Delta S^{(1)} & :=\delta\left(\frac{\partial S}{\partial \lambda}\right)_{\lambda=0}+\delta\left(\frac{\partial S}{\partial \tau}\right)_{\tau=0} \\
\Delta S^{(2)} & :=\frac{\delta^{2}}{2}\left(\frac{\partial^{2} S}{\partial \lambda^{2}}\right)_{\lambda=\tau=0}+\frac{\delta^{2}}{2}\left(\frac{\partial^{2} S}{\partial \tau^{2}}\right)_{\lambda=\tau=0}+\delta^{2}\left(\frac{\partial^{2} S}{\partial \lambda \partial \tau}\right)_{\lambda=\tau=0},
\end{aligned}
$$

and that for the stress energy is:

$$
\begin{aligned}
& T_{a b}(g(\delta), \psi(\delta))-T_{a b}(g(0), \psi(0))=\Delta T_{a b}^{(1)}+\Delta T_{a b}^{(2)} \\
& \Delta T_{a b}^{(1)}:=\delta\left(\frac{\partial T_{a b}}{\partial \lambda}\right)_{\lambda=0}+\delta\left(\frac{\partial T_{a b}}{\partial \tau}\right)_{\tau=0} \\
& \Delta T_{a b}^{(2)}:=\frac{\delta^{2}}{2}\left(\frac{\partial^{2} T_{a b}}{\partial \lambda^{2}}\right)_{\lambda=\tau=0}+\frac{\delta^{2}}{2}\left(\frac{\partial^{2} T_{a b}}{\partial \tau^{2}}\right)_{\lambda=\tau=0}+\delta^{2}\left(\frac{\partial^{2} T_{a b}}{\partial \lambda \partial \tau}\right)_{\lambda=\tau=0}
\end{aligned}
$$

Equations (4.2), (4.5) constitute the first order contribution in the smallness parameter $\delta$ to the variation of $S, T_{a b}$ so that the right hand side of these equations is identical to that of equations (3.1) and (3.2) multiplied by $\delta$.

Equations (4.3), (4.6) list the second order contributions. We note that terms which have no partial derivative(s) with respect to $\tau$ correspond to contributions from varying the geometry while keeping the state fixed. Similarly, terms which have no partial derivative(s) with respect to $\lambda$ correspond to contributions from varying the state while keeping the geometry fixed. 
Motivated by the discussion at the end of Section 3, we shall assume, for the rest of the paper that it is possible to identify state spaces on different geometries in such a way that the following holds:

A1:All 'IR' contributions to $S, T_{a b}$ in equations (4.2)- (4.6) arising from varying the geometry while keeping the state fixed can be neglected.

\section{COMMENTS RELATED TO COHERENT STATES IN THE SETTING OF REFERENCE [2]}

As mentioned in section 1, the emergence of the Einstein equations for first order variations from the proposal of maximal vacuum entanglement suggests that we enquire if this principle has anything to say about the Einstein equations for the physically interesting case of finite but small variations to coherent states. In this section we pursue this question using the estimates of the variation of $S, T_{a b}$ displayed in the previous section in equations (4.2)(4.6) for variations to coherent states described by equation (A10).

First consider the estimate to first order in the smallness parameter $\delta$ given by equation (4.2). Specializing to the coherent state case, the fact that on a fixed geometry the entanglement entropy of a coherent state is the same as that of the vacuum implies that the second term on the right hand side of equation (4.2) vanishes. As in section 3, the First Law of Entanglement entropy relates this term to the second term of equation (4.5). From section 2 and the Appendix we see that this term also vanishes by virtue of the fact that the stress energy expectation value at fixed geometry is quadratic in the parameter $\delta$. Thus there is no contradiction between the First Law of Entanglement entropy and the fact that the entanglement entropy of a coherent state is independent of its stress energy.

Note also that Jacobson's proposal of maximal vacuum entanglement entropy implies, as discussed in section 3, that the right hand side of (4.2) vanishes under the assumption A1 of section 4. This, in turn, implies the vanishing of the UV contribution to the first term of (4.2). This implies that the area of the small geodesic ball under consideration ${ }^{3}$ does not change to first order in the smallness parameter $\delta$. Finally, under the assumption A1, the first term on the right hand side of (4.5) also vanishes. Thus we conclude that the change in entanglement entropy and stress energy expectation value under finite but small variations to coherent states vanish to first order in the smallness parameter. This is consistent with the fact that under such variations (and under assumption A1), the Einstein

\footnotetext{
${ }^{3}$ At this stage it is appropriate to note that our considerations in this section are based on a single small geodesic ball centred on some fixed spacetime point and on the unperturbed maximally symmetric geometry of this ball, its associated vacuum state and coherent state excitations of this vacuum. In contrast, the principle of maximal vacuum entanglement applies to any spacetime point, the vacuum in question then being the local vacuum associated with the local patch of maximally symmetric spacetime about this point. In Reference [2], while the unvaried state is this local vacuum, the varied state is global in the sense that it is defined on the entire spacetime not just a local patch. If we choose this global varied state such that its restriction to a fixed ball about a fixed spacetime point is that of a coherent state excitation of the local vacuum in that ball, the extent to which we can continue to think of this global state as a coherent state excitation of the local vacuum as the spacetime point varies over the entire spacetime, is not clear.
} 
equations relate the non-trivial area change of the small geodesic ball to the stress energy tensor only at second order in the smallness parameter.

Let us now move on to the contributions to $S, T_{a b}$ at second order in the smallness parameter as catalogued in (4.3) and (4.6). While we would like to use these equations to derive the Einstein equations for finite, small variations to coherent states from the principle of maximal vacuum entanglement, we are unable to do so. Instead we shall use these equations to examine if the Einstein equations are consistent with the principle of maximal vacuum entanglement entropy for such variations.

The first term in equation (4.6) as well as the IR contribution to the first term in equation (4.3) can be neglected by virtue of our assumption A1. The second term in these equations contains no $\lambda$ derivative and corresponds to the contribution when the state is varied and the geometry is held fixed. In the coherent state case these contributions can be evaluated: in (4.3) this term vanishes by virtue of our considerations in section 2 and the Appendix, and in (4.6) this term yields the stress energy of the coherent state on the unperturbed geometry. From equation (A9), this term in (4.6) is exactly the classical stress energy of the classical free scalar field solution which labels the coherent state. We assume that the classical stress energy satisfies the weak energy condition so that this term is positive.

Finally, we also have the third 'mixed' terms in equations (4.3) and (4.6) and we do not know at present how to estimate them. If these terms are negligible then the only remaining contribution to (4.3) is the UV term and the only remaining contribution to (4.6) is its second term, which as argued above, is positive. Further, since (under assumption A1) the contributions (4.2), (4.5) at first order in the smallness parameter vanish, the leading order contribution to the entanglement entropy (4.1) is the UV term at second order in the smallness parameter and the leading order contribution to the stress energy (4.4) is the second term of (4.6) which is positive and also of second order.

Let us now assume that the Einstein equations hold. From Reference [2] it follows that the positive stress energy leads to an area deficit. Since the UV contribution in (4.3) is proportional to this area variation, this UV contribution is negative. Thus if the terms we have neglected can indeed be neglected and if the Taylor approximation is valid, we find that the variation of the total entanglement entropy is bounded by a negative quantity times $\delta^{2}$ so that the maximal entanglement entropy principle is consistent with the Einstein equations in the coherent state case as anticipated by Jacobson [2].

Before we end this section we would like to emphasise that coherent states can only be defined in the context of free field theory; our considerations would therefore not apply to (strongly) interacting quantum fields.

\section{COMMENTS RELATED TO THE SETTING OF REFERENCE [5]}

The proposal that Black Hole entropy be identified with entanglement entropy of quantum fields across the horizon was first made by Sorkin and his collaborators [11]. The finiteness of this entropy then relies on UV regulators. Bianchi's beautiful idea [5] is to eliminate reliance on UV physics by considering variations of entanglement entropy.

Specifically, 4 dimensional classical gravity with matter is treated as a perturbation expansion in (the square root of) Newton's constant $G$ and the theory is quantized order by order in this expansion. This quantization is then applied to the context of the 'asymptotic Rindler Horizons' defined by Jacobson and Parentani [14]. If all fields are in the vacuum state, the metric is just the flat metric with inertial coordinates $(t, x, y, z)$ and a Rindler 
wedge can be defined as the region bounded by the union of the two half hyperplanes $v=t+z=0, z \geq 0$ and $u=t-z=0, z \geq 0$. The fields are then varied about the vacuum so as to describe a stress energy pulse which hits the $u=0$ boundary of the wedge at some $t>0$. The Einstein equations imply that this stress energy perturbs the flat geometry ${ }^{4}$. A perturbed Rindler horizon can then be defined as a set of light rays which are initially divergent at $t=0=v$ and are then focussed by the matter so that they emerge collimated at future null infinity.

The first order variation of the entanglement entropy is given by equation (3.1) where the spatial region of interest, $B$, is now the half-space $t=0, z \geq 0$. The UV contribution of the first term is computed across the surface at $t=z=0$. The (expectation value) geometry of the unperturbed flat metric and the perturbed one agree to leading order in $\sqrt{8 \pi G}$ in the vicinity of this surface (see equation (6.2) below). Since the UV contribution is only sensitive to the spacetime geometry in the vicinity of this surface, one expects no ' $U V$ ' component to this variation. This is the key idea of Bianchi which allows the possibility of computing the entropy variation without recourse to UV physics.

The second term in equation (3.1) is then related to (as before, $1 / \hbar$ times an integral of) the second term of equation (3.2) through the First Law of Entanglement Entropy. ${ }^{5}$ The quantum Einstein equations at leading order in $\sqrt{G}$ (see (6.2) below) then relate this second term of equation (3.2) with the first order variation of the area of the horizon. This area variation corresponds to the first order variation of the Bekenstein Hawking entropy.

What about the possible IR contributions from the first term, of equation (3.1)? ${ }^{6}$ While an appeal to assumption A1 of section 4 allows for their neglect, we make the case for their neglect stronger by arguing below that any contributions arising from derivatives with respect to ' $\lambda$ '(i.e. from metric change) are higher order in the perturbative parameter $G$ and can hence be dropped. Our argument applies to any term with ' $\lambda$ ' derivatives in equations (3.1), (4.2) and (4.3).

The first step in the argument is to emphasise that the contribution to the entropy variation arising from the second term of equation (3.1) is independent of the perturbative expansion parameter $G$. This contribution evaluates to the area variation divided by $G$; equation (6.2) shows that the area variation itself is of order $G$ so that this variation divided by $G$ is indeed independent of $G$ (and equal to an appropriate $G$-independent integral of the stress energy via the First Law of Entanglement Entropy). The second step is to note again that the beauty of Bianchi's [5] set up is that there is no geometry change in the vicinity of the entangling surface so that one expects all contributions to the entropy variation to be UV finite. Specifically there is no dependence on a UV cutoff at the Planck Scale and hence no dependence on $G^{-1}$. Further, if we take $G \rightarrow 0$, equation (6.2) implies that there is no geometry change. Together with the UV finiteness property, this is in turn implies that any derivative of the entropy with respect to $\lambda$ must vanish in the $G \rightarrow 0$ limit.

\footnotetext{
${ }^{4}$ We shall restrict attention to a perturbation only of the matter vacuum so that the graviton is still in its vacuum.

${ }^{5}$ Reference [5] predates Reference [3] so that Bianchi derived the relation between entanglement entropy variation and stress energy independent of Reference [3].

${ }^{6}$ As in Reference [2], there is no explicit mention of possible IR contributions to the first term in equation (3.1) in Reference [5]; for our derivation of Bianchi's results to go through, these contributions should be neglegible. As shown below, this is true if $\mathbf{A 2}$ holds.
} 
To summarise: In addition to the arguments presented in support of $\mathbf{A} \mathbf{1}$, the argument above implies the statement A2:

All terms with $\lambda$ derivatives in equations (3.1), (4.2) and (4.3) yield contributions which vanish as $G \rightarrow 0$ and can be dropped in comparision with the leading order $G$-independent contribution to the entropy which is of concern to us.

In particular, this implies the neglect of IR contributions to the first term of (3.1) so that Bianchi's results on the equality of the first order variation of entanglement entropy with that of the Bekenstein- Hawking area variation follow.

One may question as to what happens when the matter is in a coherent state which is a finite, small variation about the vacuum; on the one hand it seems that one can throw stress energy through the horizon with no entropy cost but on the other, the Einstein equations mandate that any energy flux must cause area change through the Raychaudhuri equations. The entanglement entropy change, as in section 5, vanishes to first order in the smallness parameter under our assumption A2 above. As shown in section 2 the stress energy contribution to the right hand side of equation (6.2) also vanishes to to first order in the smallness parameter so that there is no area change to first order in the smallness parameter consistent with no change in the entanglement entropy. Thus with the caveats that our assumption A2 on IR contributions to equation (3.1) holds we see that there is no contradiction with the first order analysis of Reference [5].

What about an analysis to second order in the smallness parameter for coherent states? In the setting of References [5, 6], since we are using the quantum Einstein equations (to leading order in $\sqrt{G}$ ), rather than attempting to derive them as in Reference [2], we can proceed further as follows.

At the classical level, the key equations [6] which relate the 'Coulombic' part of the gravitational perturbation at leading order in $G$ to the stress energy are:

$$
\begin{aligned}
g_{\mu \nu} & =\eta_{\mu \nu}+\sqrt{8 \pi G}\left(h_{\mu \nu}^{\mathrm{rad}}+h_{\mu \nu}^{\text {source }}\right) \\
h_{\mu \nu}^{\text {source }} & =-\sqrt{8 \pi G} \int d^{4} x^{\prime} G_{R}\left(x, x^{\prime}\right)\left(T_{\mu \nu}\left(x^{\prime}\right)-\frac{1}{2} \eta_{\mu \nu} T_{\rho}^{\rho}\left(x^{\prime}\right)\right)
\end{aligned}
$$

where $h_{\mu \nu}^{\mathrm{rad}}$ is a solution to the massless wave equation in the flat spacetime, $G_{R}\left(x, x^{\prime}\right)$ is the retarded Green's function for this equation, and $T_{\mu \nu}$ is the total stress energy of the matter field $\Phi$ and the gravity wave $h_{\mu \nu}^{\mathrm{rad}}$. In the quantum theory, this stress energy is replaced by the corresponding normal ordered (with respect to the flat spacetime) operator. Since we are interested in the case where the graviton state remains in its vacuum (see Footnote 4), when we perturb the matter state away from its vacuum the expectation value metric (which we also denote by $g$ ) only gets contributions from that of the $\hat{h}_{\mu \nu}^{\text {source }}$ operator.

Let us now focus in detail on perturbations to coherent states of the matter. Accordingly we define the 1 parameter set of coherent states $\psi(\lambda):=|\lambda H\rangle$ (see equation (2.8)). Taking the expectation value of equation (6.2) in the state $\psi(\lambda)$ defines the 1 parameter family of corresponding expectation value metrics $g(\lambda)$. It follows immediately that the leading order geometry change $g(\delta)-\eta$ is second order in $\delta$, first order in $G$ and zeroth order in $\hbar$ (the latter follows from equation (2.7)).

As discussed above, the variation of the entanglement entropy vanishes (modulo assumption A2) to first order in the smallness parameter $\delta$ as does the stress energy contribution to the right hand side of (6.2). We now examine the second order contributions (4.3). The first term of (4.3) corresponds to the contribution from the varied geometry and the unvaried 
(vacuum) state. Its IR part can be neglected by our assumption A2. The UV part of the first term vanishes, similar to the vanishing of the UV part of the first term of (4.2) discussed above, because the geometry in the vicinity of the entangling surface does not change to leading order in $G$. The second term in (4.3) has no $\lambda$ derivative. Therefore, it corresponds to the second order contribution from the varied (coherent) state on the unvaried (flat) geometry. This contribution vanishes identically by virtue of the equality (see section 2) of the vacuum and coherent state entanglement entropies on the unvaried geometry. The last 'mixed derivative' term in (4.3) has a single derivative with respect to $\lambda$ and can be dropped by Assumption A2. In addition the following formal evaluation of this term using the chain rule suggest that it is not only higher order in $G$ but also vanishes identically:

$$
\left(\frac{\partial^{2} S}{\partial \lambda \partial \tau}\right)_{\lambda=\tau=0}=\left(\int \frac{d g(x)}{d \lambda}\left(\frac{\delta}{\left.\delta g_{(} x\right)}\left(\frac{\partial S}{\partial \tau}\right)\right)\right)_{\lambda=\tau=0}=0
$$

where we have used the fact (see discussion above) that the metric perturbation is of order $\delta^{2}$ so that $\left.\frac{d g(x)}{d \lambda}\right|_{\lambda=0}=0$. It is not clear to us as to what the exact range of integration should be in equation (6.3) as this depends on the details of the way in which the state $\psi(\tau)$ is identified as a state on the perturbed spacetime geometry. Nevertheless it seems reasonable that, given that the metric is unchanged to first order, any single derivative with respect to $\lambda$ vanishes when evaluated at the origin. In any case both assumption $\mathbf{A} 2$ and the formal evaluation (6.3) indicate that the mixed derivative term can be dropped.

The estimate above, finds a vanishing entanglement entropy variation when the entangling surface is the noncompact region $(z=t=0)$ and implies that the entanglement entropy per unit area of the entangling surface does not change to order $\delta^{2}$.

In contrast, as in [5], equation (6.2) can be used to relate the change in $G^{-1}$ times the area of a small part of the $t=0, z=0$ surface (representing the unperturbed cross section of the null congruence of interest) to the stress energy flux so that $G^{-1}$ times the area change is nontrivial to order $\delta^{2}$. We emphasise that, as seen from equation (6.2), the area change is itself of order $G$ so that this change divided by $G$ is independent of $G$ and proportional to $\delta^{2}$ (which is exactly the order of the coherent state stress energy on the right hand side of equation (6.2) ). Thus on the one hand the $G$-independent entanglement entropy change for coherent states vanishes to order $\delta^{2}$ and on the other, the $G$-indepenent Bekenstein Hawking entropy change is non-trivial to order $\delta^{2}$.

Hence if the formal aspects of the above arguementation (including the issue of the existence of a Taylor series expansion as envisaged in equations (4.1)- (4.3)) can be made rigorous and if assumption A2 holds ${ }^{7}$ then the ideas of [5] do not seem to apply to the coherent state example of section 2.

One viewpoint ${ }^{8}$ on this result is that coherent state excitations do not represent quasitatic, near equilibrium perturbations for which the Clausius relation " $d Q / T=d S$ " is expected to hold. If this viewpoint is correct then one may argue that the near equilibrium changes which underly black hole thermodynamics are simply not represented by coherent state perturbations. Indeed, the First Law of Entanglement Entropy relates the change in entanglement entropy ' $d S$ ' to the boost energy influx (represented by the stress energy) divided by a 'geometric' temperature proportional to $\hbar$ and may be viewed as a quantum

\footnotetext{
${ }^{7}$ See Footnote 6 in this regard.

${ }^{8}$ We thank the referee for bringing this viewpoint to our notice.
} 
version of the Clausius relation. From this point of view, coherent states do not conform to this Clausius relation and hence one does not expect black hole thermodynamics to follow.

We do not subscribe to this viewpoint for the following reasons. First, it is not clear to us that the change in black hole entropy can be accounted for by entanglement entropy change alone. If we are open to the possibility that the two are not identical, the ' $d S$ ' term in the First Law of Entanglement Entropy need not coincide with the change in thermodynamic entropy so that the latter could still be driven by the physical stress energy even if this stress energy is non-trivial only at second order. In other words we are unsure if the First Law of Entanglement Entropy is indeed a quantum version of the Clausius relation for black hole thermodynamics. Second, a coherent state excitation is as close to a classical excitation as possible. The coherent state field expectation value as well as its stress energy can be nicely confined to a region of compact support on the $t=0$ slice so as not to extend across the horizon at $t=0$. More in detail the coherent state can be modelled on a classical function which is dominated by large wavelengths and whose support is confined away from the horizon at $t=0$ and thereby, on intuitive physical grounds, constitutes a small, slowly evolving quasistatic perturbation moving towards the horizon. Further support for the nice properties of coherent states comes from the work of Ford and Kuo [4], in which it is argued that stress energy fluctuations for coherent states are minimal. On physical grounds, one would expect near equilibrium perturbations to be modeled by states which do exhibit such minimal fluctuations of stress energy and, consequently, of geometry. Indeed for a wide variety of states which constitute nontrivial first order perturbations of the vacuum and the stress energy, Ford and Kuo [4] argue that there are regions in spacetime where the stress energy fluctuations are large. Such states include single mode superpositions of the vacuum and a 2 particle state as well as single mode squeezed states which are far away from coherent states in the sense that the squeezing parameter is larger than the coherent state parameter [4]. ${ }^{9}$ In general the expectation is that a state exhibits large fluctuations of stress energy wherever the stress energy is negative [4]. Coherent states have everywhere positive energy density (for classical field theories which satisfy positive energy conditions as assumed in this work) and have minimal stress energy fluctuations everywhere. Hence in all these aspects we view them as ideal models for quasistatic near equilibrium perturbations.

To summarise: We envisage at least two different viewpoints on our result that the considerations of Reference [5] for first order variations of entanglement entropy do not seem to extend to the case of finite but small variations to coherent states. From the first point of view coherent state perturbations are simply not near equilibrium perturbations and it is no surprise that the considerations of Reference [5] do not extend to this case as this case does not represent a physically relevant near equilibrium situation. The second viewpoint asserts that coherent state perturbations modeled on suitable classical solutions can indeed be thought of as quasistatic, near equilibrium perturbations for which fluctuations in the stress energy and metric are minimal. From this point of view, our results indicate that such coherent state perturbations constitute a genuine obstruction to an extension of the ideas of Reference [5] from the case of first order variations to the case of small but finite variations.

\footnotetext{
${ }^{9}$ Single mode states extend all over spacetime and hence are not valid examples of small, confined perturbations. However, it seems reasonable to us to expect that similar results hold for confined, wave packet generalizations of such states which are dominated by some large wavelength; unfortunately, we do not know of explicit examples and it would be of interest to investigate if such examples exist.
} 


\section{Acknowledgements:}

I thank Ted Jacobson for his generous help with my numerous questions with regard to Reference [2] and for his comments on a draft version of this work. I thank Abhay Ashtekar for discussions and an anonymous referee for her/his comments.

\section{Appendix A: Coherent states in curved spacetime}

Let $(M, g)$ be a globally hyperbolic $d$-dimensional spacetime with metric $g$. Denote a Cauchy slice in $(M, g)$ by $\Sigma$ where $\Sigma$ is a $d-1$ dimensional orientable manifold without boundary. Let $\mathcal{R}_{\mathcal{I}}, \mathcal{R}_{\mathcal{I I}}$ be closed $d-1$ dimensional submanifolds of $\Sigma$ such that $\Sigma=\mathcal{R}_{\mathcal{I}} \cup \mathcal{R}_{\mathcal{I I}}$ and $\mathcal{R}_{\mathcal{I}} \cap \mathcal{R}_{\mathcal{I I}}=\mathcal{S}$ where $\mathcal{S}=\partial \mathcal{R}_{\mathcal{I}}=\partial \mathcal{R}_{\mathcal{I I}}$ is a $d-2$ dimensional hypersurface. We restrict attention to the case that $\mathcal{R}_{\mathcal{I}}$ is a small geodesic ball [2] and the case $M=R^{4}$ with $\mathcal{R}_{\mathcal{I}}$ the right half plane $z \geq 0[5]$.

Let $\Phi$ be a free scalar field propagating on $(M, g)$. We assume that the free field dynamics on $M, g$ exhibits unique evolution from initial data in any causal domain and that smooth data of compact support evolve to smooth solutions as in Lemma 3.1 of [15]. Let the initial data on $\Sigma$ be $(\phi, \pi)$ where $\phi$ denotes the scalar field on $\Sigma$ and $\pi$ its conjugate momentum. Let $\Phi$ be quantized with respect to some choice of complex structure $J$ (i.e. mode decomposition) [15] of this data on $\Sigma$ and let $|0\rangle$ be the vacuum state and $\mathcal{F}$ the Fock space, with respect to this choice. Likewise, considering $\mathcal{R}_{\mathcal{I}}$ and $\mathcal{R}_{\mathcal{I I}}$ as Cauchy slices for their domains of dependence $D_{I}, D_{I I}$, let $J_{I}, J_{I I}$ be complex structures for data on $\mathcal{R}_{\mathcal{I}}, \mathcal{R}_{\mathcal{I I}}$ with associated Fock quantizations $\mathcal{F}_{\mathcal{I}}, \mathcal{F}_{\mathcal{I I}}$.

Since the field operators $\hat{\phi}(x), \hat{\pi}(x)$ are operator valued distributions on $\Sigma$, given smooth smearing functions $e, f$ of compact support on $\Sigma$ we have that the operator $\hat{U}(e, f)$ is unitary where

$$
\hat{U}(e, f):=e^{i \int_{\Sigma} f(x) \hat{\phi}(x)-e(x) \hat{\pi}(x)}
$$

Define the coherent state $|e, f\rangle$ as

$$
|e, f\rangle:=\hat{U}(e, f)|0\rangle
$$

Define $\hat{\rho}_{I}(e, f)=\operatorname{Tr}_{\mathcal{F}_{\mathcal{I I}}}(|e, f\rangle\langle e, f|)$ so that $\hat{\rho}_{I}(0,0)=\operatorname{Tr}_{\mathcal{F}_{\mathcal{I I}}}(|0\rangle\langle 0|)$. We argue below that the entanglement entropy $S(e, f)=-\operatorname{Tr}_{\mathcal{F}_{\mathcal{I}}}\left(\hat{\rho}_{I}(e, f) \ln \hat{\rho}_{I}(e, f)\right)$ is the same as the vacuum entanglement entropy $S(0,0)=-\operatorname{Tr}_{\mathcal{F}_{\mathcal{I}}}\left(\hat{\rho}_{I}(0,0) \ln \hat{\rho}_{I}(0,0)\right)$ modulo issues of UV divergences.

Define

$$
\begin{aligned}
& \hat{U}_{I}\left(e_{I}, f_{I}\right)=e^{i \int_{\mathcal{R}_{\mathcal{I}}} f_{I}(x) \phi(x)+e_{I}(x) \pi(x)}, \\
& e_{I}=e \quad \text { on } \quad \mathcal{R}_{\mathcal{I}} \quad e_{I}=0 \quad \text { on } \quad \mathcal{R}_{\mathcal{I I}} \text {. } \\
& f_{I}=f \quad \text { on } \quad \mathcal{R}_{\mathcal{I}} \quad f_{I}=0 \quad \text { on } \quad \mathcal{R}_{\mathcal{I I}} .
\end{aligned}
$$

and likewise for $I \leftrightarrow I I$.

Let $e, f$ be supported away from $\mathcal{S}$. Then we have that $\hat{U}_{I}\left(e_{I}, f_{I}\right)$ and $\hat{U}_{I I}\left(e_{I I}, f_{I I}\right)$ are unitary operators on $\mathcal{F}, \mathcal{F}_{\mathcal{I}}$ and $\mathcal{F}, \mathcal{F}_{\mathcal{I I}}$ respectively and that

$$
\hat{U}(e, f)=\hat{U}_{I I}\left(e_{I I}, f_{I I}\right) \hat{U}_{I}\left(e_{I}, f_{I}\right)=\hat{U}_{I}\left(e_{I}, f_{I}\right) \hat{U}_{I I}\left(e_{I I}, f_{I I}\right)
$$


We have that

$$
\begin{aligned}
\hat{\rho}_{I}(e, f) & =\operatorname{Tr}_{\mathcal{F}_{\mathcal{I I}}}\left(\hat{U}_{I I}\left(e_{I I}, f_{I I}\right) \hat{U}_{I}\left(e_{I}, f_{I}\right)|0\rangle\langle 0| \hat{U}_{I}\left(e_{I}, f_{I}\right)^{\dagger} \hat{U}_{I I}\left(e_{I I}, f_{I I}\right)^{\dagger}\right) \\
& =\operatorname{Tr}_{\mathcal{F}_{\mathcal{I I}}}\left(\hat{U}_{I}\left(e_{I}, f_{I}\right)|0\rangle\langle 0| \hat{U}_{I}\left(e_{I}, f_{I}\right)^{\dagger}\right)=\hat{U}_{I}\left(e_{I}, f_{I}\right) \operatorname{Tr}_{\mathcal{F}_{\mathcal{I I}}}(|0\rangle\langle 0|) \hat{U}_{I}\left(e_{I}, f_{I}\right)^{\dagger}
\end{aligned}
$$

where we have used the invariance of the Trace under unitary tranformations, and the fact that $U_{I}(e, f)$ acts as the identity operator on $\mathcal{F}_{\mathcal{I I}}$ in the last line. The equality of $S(e, f)$ and $S(0,0)$ then follows, once again from the invariance of the Trace under unitary transformations. Our demonstration suffers from exactly the same UV divergences as in the case of flat spacetime discussed in section 2 . That discussion clearly applies here and, while we do not provide an explicit regularization, indicates the any UV regulation softens the sharp boundary $\mathcal{S}$ and strongly suggests that the above argument can be made well defined.

Next, let the vacuum state be Hadamard. It is straightforward to check, for example using sections 3,4 of [15], that the two point function in the coherent state $|e, f\rangle$ evaluates to

$$
\left\langle e, f\left|\hat{\Phi}(X) \hat{\Phi}\left(X^{\prime}\right)\right| e, f\right\rangle=\left\langle 0\left|\hat{\Phi}(X) \hat{\Phi}\left(X^{\prime}\right)\right| 0\right\rangle+H(X) H\left(X^{\prime}\right)
$$

where $H$ is scalar field solution obtained from the initial data $(e=\phi(x), f=\pi(x)), x \in \Sigma$ and $H(X), H\left(X^{\prime}\right)$ are the values of $H$ at the spacetime points $X, X^{\prime} \in M$. From our assumptions on free field evolution it follows that $H$ is smooth. Equation (A9) then implies that $|e, f\rangle$ is also Hadamard for any choice of smooth and compactly supported $(e, f)$. Since we are interested in stress energy expectation value relative to the vacuum and since the stress energy operator is quadratic in the field, we adopt a normal ordering prescription with respect to our choice of annihilation and creation operators on $\mathcal{F}$. We are interested in coherent states which are first order departures from the vacuum. For some small positive parameter $\delta$, consider the coherent state $|\delta e, \delta f\rangle$ so that

$$
|\delta e, \delta f\rangle=e^{i \delta \int_{\Sigma} f(x) \hat{\phi}(x)-e(x) \hat{\pi}(x)}|0\rangle
$$

Expanding the exponential out and noting that the field operators are linear in the annihilation- creation modes, we obtain an expansion of the coherent state in terms of $n$ - particle states with the norm of the $m$-particle component of order $\delta^{m}$. In particular the one particle component is of order $\delta$ so that this coherent state constitutes a first order variation from the vacuum. Since the (normal ordered) stress energy tensor is quadratic in the field operators, the order $\delta$ contribution to its expectation value which is from the off diagonal matrix element between its vacuum component and its 1 particle component, vanishes, so that the leading order contribution is at second order ${ }^{10}$

More generally, since the stress energy operator is quadratic in the modes, the only way for any state to be both a first order variation of the vacuum and exhibit a non-trivial normal ordered stress energy expectation value at first order is if its two particle component is of

10 This can also be seen directly from the expression for the two point function (A9) which implies that the stress energy expectation value is exactly that of the classical solution $\delta \times H$. 
order $\delta$.

[1] T. Jacobson, Phys.Rev.Lett. 75 (1995) 1260-1263

[2] T. Jacobson e-Print: arXiv:1505.04753 [gr-qc]

[3] T. Faulkner, M. Guica, T. Hartman, R. C. Myers and M. Van Raamsdonk, JHEP 1403 (2014) 051; D. Blanco, H. Casini, L-Y Hung and R. C. Myers, JHEP1308 (2013) 060

[4] C-I. Kuo and L. Ford, Phys.Rev. D47 (1993) 4510-4519

[5] E. Bianchi, arXiv:1211.0522 [gr-qc]

[6] E. Bianchi and A. Satz, Phys.Rev. D87 (2013) 124031

[7] T. M. Fiola, J. Preskill, A. Strominger and S. Trivedi, Phys.Rev. D50 (1994) 3987-4014

[8] E. Benedict and S-Y Pi Annals Phys.245 (1996) 209-224

[9] S. Das and S. Shankaranarayanan, Phys.Rev. D73 (2006) 121701

[10] H. Casini, D. A. Galante and R. C. Myers e-Print: arXiv:1601.00528

[11] R.D. Sorkin in Tenth International Conference on General Relativity and Gravitation Vol. II, 734-736 (1983) edited by B. Bertotti, F. de Felice and A. Pascolin, also available as arXiv:1402.3589 [gr-qc]; L. Bombelli, R. K. Koul, J. Lee and R. D. Sorkin, Phys.Rev. D34 (1986) 373-383

[12] M. Srednicki, Phys.Rev.Lett.71 (1993) 666-669

[13] S.W. Hawking, Commun.Math.Phys. 43 (1975) 199-220

[14] T. Jacobson and R. Parentani, Found.Phys. 33 (2003) 323-348

[15] R. Wald, Quantum Field Theory in Curved Spacetime and Black Hole Thermodynamics (The University of Chicago Press 1994) 